\title{
Using Bayesian networks to quantify the reliability of a subsea system in the early design
}

\author{
J. Zhang, Y. Liu \& M.A. Lundteigen \\ Department of Production and Quality Engineering, Norwegian University of Science and Technology, \\ Trondheim, Norway \\ L. Bouillaut \\ French Institute of Science and Technology for Transport, Development and Networks, \\ Marne-la-Vallée Cedex, France
}

\begin{abstract}
Quantification of the reliability of systems is an essential task when evaluating new technologies, since a lack of adequate reliability performance will violate the intended gain of the innovation. Several models for reliability assessment have been proposed in literature. However, they are often criticized for not being very useful in early evaluations of new design concepts, as they may not be able to include new operating aspects in the models, such as new ways of operating and new environmental exposures. Bayesian formalism, as a probabilistic modeling approach, is experiencing a growing success due to its flexibility in modelling various system features. This paper reviews the valuable features offered by Bayesian formalism, and explores the possible advantages of using Bayesian Networks for reliability assessment in the early design phase of subsea systems. The applicability of adapting Bayesian formalism for this purpose has been demonstrated using a high integrity pressure protection system installed on the seabed to protect a hydrocarbon pipeline against overpressures.
\end{abstract}

\section{INTRODUCTION}

Reliability is one of the key performance measures of technical systems used to demonstrate the ability of the system to carry out the desired function over time (Rausand \& Høyland, 2004). The reliability of a structured system can be evaluated by using the suitable modelling approach to show how the potential events (e.g. component failure, maintenance and testing) can influence the system failure. The quantification of reliability can form as a basis for decision-making concerning different stages of the system development process (i.e. design, construction and operation and maintenance) (Rausand, 2014).

An overview of modelling approaches available to quantify reliability may be found in literature (Rausand, 2014; Rausand \& Høyland, 2004). However, none of the modelling approaches can fit for all types of systems, especially when the operational philosophies of the selected system are complex and the associated effect remains dormant to analysts at the early stage.

Reliability influencing factor (RIF) can represent conditions that have impact on the loss of system performance, e.g. test and maintenance strategies, human and organizational factors (HOFs), environmental factors and so on (Lundteigen \& Rausand, 2010). All relevant RIFs can in principle be included in the reliability model, but the precision in the calculated result may not necessarily be very high if the data is uncertain or not available, or invalid assumptions are made in the model(s). In practice, it seems more feasible to build a model that accounts for the most important factors instead of considering all factors of relevance with low-quality data input. This is especially the case when assessing reliability of a new (unproven) technology or system in the early stage of the design, where the details of the system have not yet been settled and few data are available.

The subsea oil and gas industry is one example of an industrial sector where innovations are needed to reduce costs and to meet stricter safety requirements. The industry is conducting a high number of reliability assessments, but experience indicate that they are carried out too late to have an effect on early design selections and decisions. To support the need to use reliability assessments more actively in the early verification of new subsea design concepts, it is necessary to develop reliability modelling approaches that can capture the most important characteristics of systems performance in its (new) operating environment, and the most important effects of uncertainty associated with these.

The objective of this paper is to adapt Bayesian formalism in reliability assessment in the early design phase, and to demonstrate how it can be applied for an oil and gas related safety system to be installed subsea. The outline of the paper is shown as follows: Section 2 introduces the basic concept of modelling approach in the reliability assessment, and points out challenges of developing feasible reliability model in light of subsea systems. Section 3 briefly reviews basic features 
of Bayesain Networks and explore the possible use in the reliability assessment for subsea systems. The applicability of proposed approach is illustrated by an example of subsea high integrity pressure protection system in Section 4. In the end, the discussion and conclusion are presented in Section 5.

\section{CHALLENGES IN MODELLING SUBSEA RELIABILITY}

The term model is always an abstraction of the reality of a real system (Long, 2012). A model can be used to qualitatively express functions in a system and with surroundings, or quantify a suitable measure of a specific system performance. The focus of this paper is only placed on the quantitative model to estimate the reliability of a structured system, which is built up on a basis of a logic model to study how the system fails, within input parameters (i.e. the failure data for selected failures). An overview of models used for reliability assessment can be found in many textbooks and standards. For safety-instrumented systems (SIS) that are required to perform their intended function upon demand, the useful reference are part 6 of IEC 61508 (2010) and ISO/TR12489 (2013), and the limitation and the application of these models can be found in a number of literatures (Innal, 2008; Johansson, 2013; Rausand \& Høyland, 2004). Most of current models for reliability or availability assessment (if downtime associated with e.g. repairs of system are included) focus on describing how the state of system changes in certain of events, such as failure, testing, repair and so forth. The probabilistic distribution is used to describe the occurrence of the event, such as failures of component which by definition we don't know when will it happen.

The term failure can be interpreted differently according to performance characteristics of systems. The success/failure of system performance is relatively easy to define within yes/no decision boundary, such as the safety function. However, developing the reliability model for the system with variable performance characteristic, requires several attempts to clearly determine unacceptable levels (or failure) of system performance (MIL-HDBK-338B, 1998). It is especially the case for subsea production and processing system where the difficulty of mitigating failures subsea is much higher than topside due to limited and costly access. This situation calls for alternative ways or 'soft means' to maintain reliability performance above the limits of acceptable performance over time, and the corresponding reliability model should therefore encounter for degraded mode of operation. However, some static models such as Fault Tree Analysis (FTA) and Reliability Block Diagram (RBD) will not be able (at least in an easy way) to model the degraded operation. Moreover, basic events in the standard FTA are statistically independent, meaning that dependencies between failures are impossible to address in standard fault tree (Bobbio, Portinale, Minichino, \& Ciancamerla, 2001).
Many systems installed on the seabed also involve dynamic system behaviors because of the complex way of operating. Some models such as Markov analysis (MA) and Petri Nets (PN) are able to give a realistic picture about dynamic features of systems in case of certain events (Rausand, 2014). However, the model based on Markov property are often criticized for the exponentially increasing size of model when modelling the system with a high level of complexity. PN may be recommended when there is a necessity to consider operational aspects such as maintenance, but it is hard to develop PN and even more hard to update the PN model when more details of system is given.

The selection of reliability model does not only depend on the type of systems, but also the stage of its development. As of today, the oil and gas industry is frequently using qualitative models (e.g. FTA and RBD are used as structure analysis) in the early design phase, and the more advanced modelling approaches are often pursued in the later stage and they are used for verification and not for design evaluation as the possibilities to influence the design is limited at this stage (equipment already ordered, decisions about technical solution taken). The use of quantitative models in early phase may also be criticized due to a lack of suitable data and details/information of system operation (Johansson, 2013). Many of the future developments in subsea require adaption of new technology and new ways of operating, however, may involve uncertainty in many aspects. For reliability assessment, the uncertainty can be categorized as model uncertainty, data/parameter uncertainty and completeness uncertainty. As the limited knowledge about the new system becoming one particular issue for early design, the completeness uncertainty is of greatest importance, followed by model and data uncertainty (Jin, Lundteigen, \& Rausand, 2012). The uncertainty should be addressed in the early evaluation to avoid the situation that too conservative design is selected to compensate for the uncertainty caused by unfamiliar operating conditions and a lack of historic performance in the beginning of development process.

Therefore, models used as basis for reliability assessment of subsea systems, also for use in the early design phase, should therefore address foreseeable situations where operation in degraded mode is required, the complex operational phenomenon, and incorporate the result of simulation (in an early design phase) as the reliability data under uncertainty. However, the classical reliability modelling approaches do not suffice for this purpose. This paper will discuss valuable features offered by Bayesian Networks, and explore the possible use for reliability assessment in the early design phase of subsea systems.

\section{BAYESIAN NETWORKS}

\subsection{Basic features of Bayesian Networks}

Bayesian Networks (BN), are used in many engineering or science disciplines since their emergence, such 
as artificial intelligence development and the decisionmaking strategy. This formalism has been recently introduced in field of reliability, availability and maintainability (RAM) analysis and experienced a growing success because of its flexibility in modelling various system features. This modelling approach, based on the Bayesian theory, can be used as a better alternative to FTA as the restrictive assumptions of FTA can be removed and dependencies between failures are incorporated in BN model (Bobbio et al., 2001). The BN model can also build up the cause-effect relationships between the multi-state variables, e.g. failure rate of a system and associated contributing factors (Jones, Jenkinson, Yang, \& Wang, 2010). Many other applications of the BN formalism can also be found in the past decade literature, proving its ability to model reliability and maintenance strategies, see (Cai et al., 2013; Cai et al., 2012).

$\mathrm{BN}$ can be expressed as a graphical representation which consists of Directed Acyclic Graph (DAG) formed by variables together with the directed edges, and Conditional Probability Tables (CPT) assigned the conditional dependencies between variables (Jensen, 1996). When a link connects a node A to another node $\mathrm{B}, \mathrm{A}$ is a parent of $\mathrm{B}$ and the variables that the two nodes denote are conditionally dependent. If the node A has not any parent, it is called as a root node and its prior probability should be specified in the CPT. The joint probability distribution of a set of variables $\left[X_{1}, X_{2} \ldots X_{\mathrm{n}}\right]$ is given as follows (Jensen, 1996), where $P a\left(X_{\mathrm{i}}\right)$ refers to the parent of $X_{\mathrm{i}}$ :

$\operatorname{Pr}\left[X_{1}, X_{2} \ldots X_{n}\right]=\prod_{i=1}^{n} \operatorname{Pr}\left[X_{i} \mid \operatorname{Pa}\left(X_{i}\right)\right]$

One of the most unique ability of $\mathrm{BN}$ is to compute the posterior probability of any nodes when the observation of a set of variable $E$, called as evidence is given. The prior probability can therefore be updated by taking advantages of Bayes' theorem (Khakzada, Khana, \& Amyotte, 2013):

$\operatorname{Pr}(U \mid E) \operatorname{Pr}(E)=\operatorname{Pr}(E \mid U) \operatorname{Pr}(U)$

\subsection{Bayesian Networks in reliability assessment}

The valuable features offered by using BN model have already been discussed by some researchers, see e.g. (Bobbio et al., 2001; Jones et al., 2010; Khakzada et al., 2013; Rausand \& Høyland, 2004). Some key factors driving the implementation of Bayesian formalism in reliability assessment can be summerized by comparing to the most widespread modelling approach in reliabiltity assement, i.e. FTA.

The states of variables being modelled in $\mathrm{BN}$ do not have to be binary as for FTA, so that the multi-states variables can be easily accommodated. The standard FTA has to connect the variables/events through a specified logic gates (i.e. AND-gate and OR-gate). This issue can be solved by using some advanced FTA tooling (e.g. dynamic fault tree) by including some other type of gates, see (Durga Rao et al., 2009). While

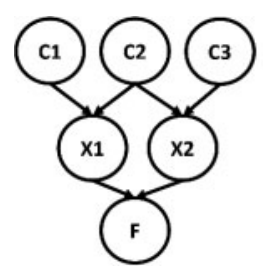

(a)

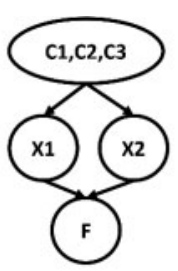

(b)

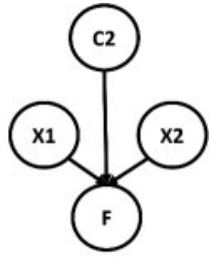

(c)
Figure 1. (a) Uncorrelated causes, (b) Correlated cause and (c) Common cause $\mathrm{C}_{2}$.

for BN models, it is possible to involve probabilistic gates, which are able to develop the complicated cause-effect relationship between variables, e.g. the failure and failure causes, the failure causes and the contributing factors.

The statistical dependencies between variables can be easily accommodated and visualized in the BN models by modifying the CPT and adding the causal arcs to connect variables. For example, in a fault tree common because failures (CCFs) and individual failures are assumed be necessarily independent, but such assumption is not needed in a BN model. In FTA, a CCF can be treated explicitly as the single input to the system failure by adding an OR-gate, or the CCF can be treated implicitly by considering it as a minimal cut set. In a BN model, a CCF can be modeled by identifying the relationships between failure causes. As shown in Figure 1, where $C_{i}$ stands for the cause that leads to the failure of component $\mathrm{X}_{\mathrm{j}}$ (connected by causal arc) and $\mathrm{F}$ stands for state of system consists of component $\mathrm{X}_{\mathrm{j}}$. In the Figure $1(\mathrm{a})$, the root variable $\mathrm{C}_{\mathrm{i}}$ are uncorrelated so that only $\mathrm{C}_{2}$ act as the $\mathrm{CCF}$ that can lead to the failure of both $\mathrm{X}_{\mathrm{j}}$. In this case, we can modify the Figure 1 (a) to Figure 1 (c), treating the CCF as one direct input to the system failure. Figure 1 (b), the root node consists of all the correlated causes so that the joint probability for all $\mathrm{C}_{i}$ should be specified in the CPT, which can avoid incorrect inclusion of dependent common causes.

Besides above, the ability to update estimation according to new information (e.g. failure rate of components or reliability of selected systems) makes BN model an appealing candidate for reliability assessment in the later phase of system development. It can be used to update estimates based on the data derived from the site acceptance testing (SAT). The detailed discussion about updating procedures using influencing algorithm within cumulative collection of occurrence over a certain interval can be found in (Khakzada et al., 2013). The updating technique can also be used in the operational phase, to forecast the change in trends that may suggest a variation in estimated reliability, based on monitoring technical states and process parameters of critical components (e.g. conditional monitoring or even online monitoring). Some similar works have already been done in the domain of risk analysis, see (Vatn, 2013). In this paper, we will study the suitability of using $\mathrm{BN}$ in the early design phase. 


\subsection{Quantifying reliability of subsea systems with $B N$}

An interesting possibility is to take advantages of Bayesian formalism to provide an approximate indication of reliability achievement of subsea innovation at the early stage, which (at least) includes the following aspects:

1) Degraded mode of operation

2) Foreseeable operational conditions

3) Flexible inclusion of RIFs

\subsubsection{Degraded mode of operation}

The variable performance characteristics can be expressed as discrete nodes in the BN. As discussed above, the operators of subsea system usually want to continue operation in case of certain type of failures, meaning a reduction in information or performance. Once the acceptable level of performance is clearly determined, subsea components/systems can be in one of the following states: (i) fully (perfectly) working state, (ii) degraded working state where the components/systems work at the reduced level but above the limit and (iii) faulty state where the performance of components/systems is considered unsatisfactory. Even for the safety system that only includes go/nogo performance attributes, the number of states in the variable can be more than two, depending on the level of redundancy. For instance, a two-of-three voted system can have three states expressed as [fully working (3oo3), degarded working (2oo3), faulty (1003 or $0 \mathrm{oo} 3)]$.

\subsubsection{Foreseeable operational conditions}

In subsea applications, known systems or technologies may be exposed to unfamiliar failure causes due to changes of operating environment and novelty itself. The impact of failure causes cannot be fully revealed based on historical data in the early design phase of new subsea application. Using probabilistic gates instead of logic gates can illustrate the relationship between the failure and its causes, and components are allowed to response differently to one particular failure cause. The uncertainty about unknown or unfamiliar relationship between failure causes and failures can therefore be outlined in the calculated result. For reliability assessment in the early design, the effects of foreseeable operational conditions will be unknown or uncertain, but the BN model can allow their inclusion while relying heavily on the other type of information (e.g. expert judgment, the relevance between industrial sectors). Therefore, the best estimates of uncertainty should be taken into account.

\subsubsection{Flexiable inclusion of RIFs}

The failure rate of component is an essential parameter input of reliability model, and it can be correspondingly assigned as the prior probability for the failure of each component in BN model. The estimation of failure rate for new equipment may be on the basis of evaluating relevant RIFs, see e.g. (Brissaud, Charpentier, Fouladirad, Barros, \& Bérenguer, 2010; Rahimi \&
Rausand, 2013). BN may allow a more flexible inclusion of RIFs, in light of following topics for failure rate estimation:

\section{- Selection of RIFs:}

The list of RIFs may vary depending on types of systems and their intended application areas. Some generic RIFs can be found in (Brissaud et al., 2010). The RIFs of subsea systems should be collected based on the expert opinions, experience from existing subsea application and recommendations from stakeholders. Normally the RIFs are selected as disjoint as possible since linear relationship are often assumed between RIFs and failure causes (Rahimi \& Rausand, 2013). However, the selected RIFs can be disjoint or correlated as dependencies between variables can be easily accomadated in Bayesian formalism.

\section{- Assign values of RIFs:}

Some RIFs like temperature are directly related to a measurement (e.g. the measured or foreseen value), but other RIFs cannot be easily measured, such as HOF or maintenance strategies. This paper tacitly assumes that RIFs can be treated as the stochastic variables in BN, meaning that all RIFs can be updated and estimated based on the mutual information (e.g. indicators, failure propagation and historical events).

According to the Bayesian philosophy, a random variable $A$, with some density function of $f(A)$ that can express what one thinks about the occurring value of $A$, before any evidence are obtained (Rausand \& Høyland, 2004). Therefore, it is possible to account for the effect of uncertainty by allocating suitable probability distribution to the variables, for example, the beta distribution for continuous variables (Vatn, 2013). If one variable $A$ in a binomial distribution is beta distributed within prior shape parameter $\alpha_{0}$ and $\beta_{0}$, the posterior probability of $A$ is still beta-distributed within posterior shape parameter $\alpha_{0}+\mathrm{s}$ and $\beta_{0}+\mathrm{n}-\mathrm{s}$, where $s$ denotes the number of $n$ trials that have outcome as outcome $X$. In this paper, only the discrete nodes are used to represent RIFs for calculation convenience.

\section{- Connecting RIFs to failure causes}

The influencing functions between RIFs and their child nodes (i.e. failure causes) can be determinted by building up the cause-effect relationship probabilistically. This is essentially based on expert judgement and system/function analysis. A high degree of uncertainty may therefore dominate the results of the reliability assessment due to biased judgement. One possible solution to overcome this obstacle in the $\mathrm{BN}$ model is to introduce different experts as a root node connecting to the failure causes, where the priors of node 'expert' are the weights of each expert. Therefore, failure-derived data can be used to adjust the weights of experts.

\section{EXAMPLE}

The subsea production and processing system faces a number of challenges in evaluating reliability of 


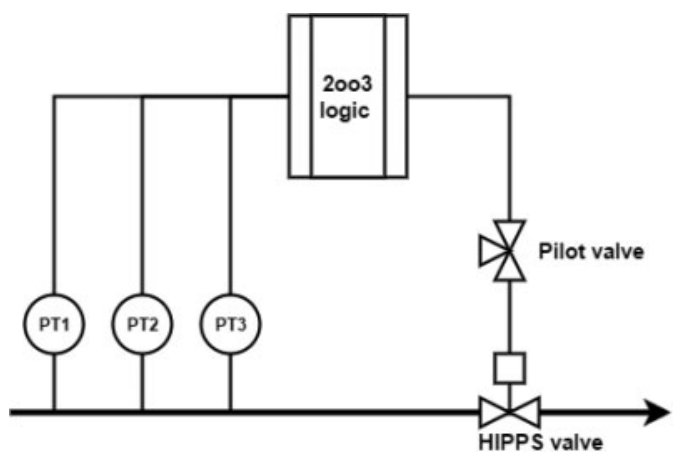

Figure 2. schematic of HIPPS functions.

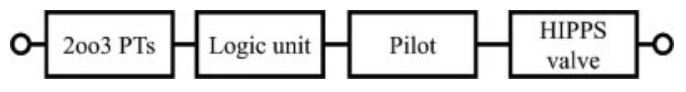

Figure 3. RBD for HIPPS function.

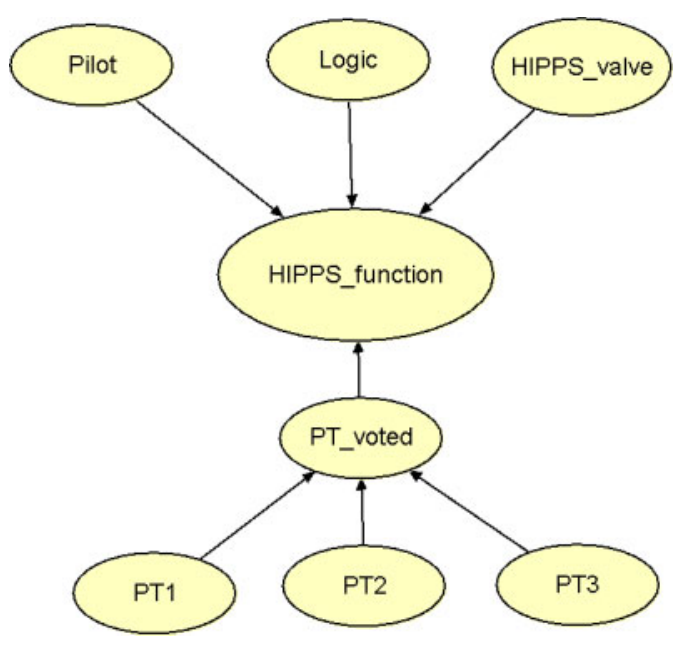

Figure 4. BN model for HIPPS function.

subsea units as they are installed in a harsh and unfamiliar working environment. This section demonstratse the applicability of proposed approach by modelling a specific failure phenomena that influence the performance of system installed subsea. This type of system is not new, but we can foresee that new type of equipment is introduced (e.g., for sensors) to enhance reliability. The computation and graphical representation of $\mathrm{BN}$ model is done by the software HUGIN (2015).

\subsection{System description}

A high integrity pressure protection system (HIPPS) is normally combined with process shutdown system to protect the downstream equipment from the overpressure. The schematic of HIPPS is illustrated in Figure 2.

The HIPPS is a typical SIS that can be divided into three modules: (i) a two-out-of-three (2003) voted
Table 1. Failure rate and prior probability of root variables.

\begin{tabular}{lll}
\hline Root variables & $\begin{array}{l}\text { Failure rate } \\
\text { (per hour) }\end{array}$ & Prior probability \\
\hline PT & $0.3 \times 10^{-6}$ & $1.314 \times 10^{-3}$ \\
Logic & $0.1 \times 10^{-6}$ & $0.438 \times 10^{-3}$ \\
Pilot & $0.8 \times 10^{-6}$ & $3.504 \times 10^{-3}$ \\
HIPPS valve & $2.1 \times 10^{-6}$ & $9.198 \times 10^{-3}$ \\
\hline
\end{tabular}

pressure transmitter (PT) system as a sensor module, (ii) a logic module, and (iii) a HIPPS valve that equipped with pilot valve as the final element module to stop the flow from upstream to downstream facilities under overpressure situation. The RBD of the HIPPS function is illustrated in Figure 3. The BN model in Figure 4 can be constructed on basis of RBD, where probability of system failure is the prior probability of variable 'HIPPS_function'. The 2003 voted system means that the system is able to respond when at least two PTs are functioning. The 2003 voted system in RBD model is considered as binary variable, whereas three states are assigned for this variable in $\mathrm{BN}$ model according to the discussion in section 3.3.1.

Table 1 lists the associated failure rate and prior probability of each components, based on the data provided in PDS data handbook (SINTEF, 2010). Since the demand rate of HIPPS is lower than once per year, the average probability of failure on demand (PFDavg) is selected as the measure of reliability as suggested by IEC61508 (2010). The PFDavg (priors in Table) can be calculated based on the failure rate $\lambda$ of each component and the test interval $\tau$ (i.e. 1 year $=8760$ hours) as follows:

$P F D_{\text {avg }}=\lambda \times \tau / 2$

The PFDavg for the HIPPS function of BN model is calculated as 0.013107 according to Equation (1), which is the same as the result of RBD since assuming that the degraded working state has the same effect as fully working state on the failure of HIPPS function. Another advantage offered by using BN model is to obtain the criticality of components by finding Most Probable Explanation (MPE) in BN model. It computes the probability of most likely configuration that leads to the system failure when the evidence is given. In this case, if the failure of HIPPS function is obseverd, the most likely explanation is determinted to be the failure of HIPPS valve, provided that other components can respond on demand. This could be explained as HIPPS valve has the highest failure rate and is connected in series.

This BN model can be itegrated with the Markov process if the repair action is taken into account to calculate the availability, where priors will be replaced by the steady-state probabilities of the corresponding states.

\subsection{Effects of subsea sensors drift}

The importance of condition monitoring that normally performed by sensors is essential to foresee failures 
under development and to make optimal interventions based on the prediction of remaining useful life. However, the industry has experienced that some sensors installed subsea are vulnerable to drift, an effect that will lead to reading offsets or the erratic reading of sensors. This may be a concern also for new sensors, despite new technology proposal to overcome this problem In topside (dry) environment, the negative impact of sensor drift could be removed by some maintenance tasks like re-calibrations, but this is not possible subsea without retrieving the sensor. In this example, the sensor drift is considered as a contributing factor that can influence the success of 2003 voted system within different magnitudes, i.e. High, Medium and Low.

Various factors can influence the magnitude of the sensor drift, such as physical property of the sensors (e.g. usage) and various environmental factors (e.g. temperature and pressure). However, the causerelationship between these subsea RIFs and sensor drift has not yet been fully captured in the subsea environment, as RIFs may vary with different design alternatives and operating environment. In this example, we tacitly assume that two RIFs, namely as 'RIF1' and 'RIF2', are relevant in estimating magnitude of drift of sensors.

In order to model this long term but slow degradation effect, some relevant assumptions need to be made as follows:

- The sensor drift introduced here is considered as the cause to the failure of PT voted system. This may be present in all three PTs at the same time, but the degree of drift can be different Therefore, the number of functioning sensors can influence the probability of responding to a high pressure condition, meaning that fully working state and degraded working state have different impact on the system failure.

- The sensor drift starts after installation, and sensors will experience different levels of drift during each test interval. In this example, the sensor drift is assumed as discrete distributed in the early evaluation

- The re-calibration may be done by software implemented compensation, using e.g. models ("virtual/soft sensors") combined with other physical measurements. But these modeling aspects of this option has not been included in the model here.

- The two RIFs can be disjoint (e.g. physical property (material) of sensors and temperature) or correlated (temperature and pressure). The statistical dependencies between selected RIFs can be incorporated according to Figure 1. In this example, the two RIFs are assumed to be disjoint. It is worth noting that the selected RIF can also connect to other nodes and such conditional dependencies can be easily accommodated in Bayesian formalism, e.g. material selection of sensors and failure rate of sensors.

The BN model that includes the sensor drift and associated RIFs is shown in Figure 5. The conditional
Table 2. Conditional probability between 'drift' and 'PT_voted'.

\begin{tabular}{|c|c|c|c|}
\hline & \multicolumn{3}{|l|}{ Drift } \\
\hline & High & Medium & Low \\
\hline Degraded working & 0.015 & 0.01 & 0.002 \\
\hline Fully working & 0.01 & 0.005 & 0.001 \\
\hline
\end{tabular}

*The values in this table are assigned for illustrative purpose

dependencies between variable 'drift' and 'PT_voted' are presented in Table 2, where values of state 'faulty' of 'PT_voted'for all states of 'drift' are assigned as 0 then can be omitted. The value assigned in Table 2 can be explained as: the 2003 voted system has a probability of 0.015 to fail in the situation that only two PTs can respond and the effect of drifting is high. Table 3 contains the conditional dependencies between two disjoint RIFs and variable 'drift'. Note that $\mathrm{H}, \mathrm{M}$, and $\mathrm{L}$ stands for states of drift effect and $-1,0,+1$ of RIFs means the associated RIF has negative effect, no effect, positive effect on the drifting. The value assigned in Table 3 can be explained as: the distribution of different drifting effect is estimated as [0.4 (High), 0.35 (Medium), 0.25 (Low)] under the situation that RIF1 has negative effect and RIF2 has positive effect. The values assumed in Table 2 and Table 3 in this example are only for the purpose of illustration.

The PFDavg of HIPPS function is now slightly increasing from 0.013107 to 0.015345 after introducing sensor drift. For this case study, if the failure of HIPPS function is obseverd, according to the result of MPE, the HIPPS-valve is the most likely one to be blamed. Therefore, one may conclude that: when the subsea HIPPS is influenced by sensor drift that is estimated in this example, the most vulnerable component is still the HIPPS valve until sensor drift reaches the pre-defined acceptable limit.

In this example, the values are assigned for the purpose of illustration. The priors of RIF1 and RIF2 are given as $[-1(0.1), 0(0.9),+1(0.1)]$ and $[+1(0.83)$, $-1(0.17)]$, expressing what one (the expert) thinks about the probabilities of states of RIFs. The priors of RIFs can be determinted based on multiple source of information, e.g. (new) interpretation of historical evidences and operation experience. The values of RIFs be continuously updated if the new information is available, e.g. the (early) simulation result. If the failure of HIPPS function is observed during the test interval, the posterior state of RIF1 and RIF2 will be updated to $[-1(0.08), 0(0.82),+1(0.1)]$ and $[+1(0.42),-1(0.58)]$ representively, according to the Equation (2). Once the new RIF/failure cause/failure mode is revealed in the later phase (e.g. the prototype testing), it can be easily merged with the existing BN models by adding the casual arc or variables. 
Table 3. Conditional probability betweenm 'RIFs' and 'drift'.

\begin{tabular}{lllllll}
\hline RIF1 & \multicolumn{2}{c}{$-1(0.1)$} & \multicolumn{2}{c}{$0(0.9)$} & \multicolumn{2}{c}{$+1(0.1)$} \\
\hline RIF2 & +1 & -1 & +1 & -1 & +1 & -1 \\
& $(0.83)$ & $(0.17)$ & $(0.83)$ & $(0.17)$ & $(0.83)$ & $(0.17)$ \\
H & 0.4 & 0.1 & 0.15 & 0.05 & 0.1 & 0 \\
M & 0.35 & 0.2 & 0.05 & 0.1 & 0.15 & 0.01 \\
L & 0.25 & 0.7 & 0.8 & 0.85 & 0.75 & 0.99 \\
\hline
\end{tabular}

*The values in this table are assigned for illustrative purpose

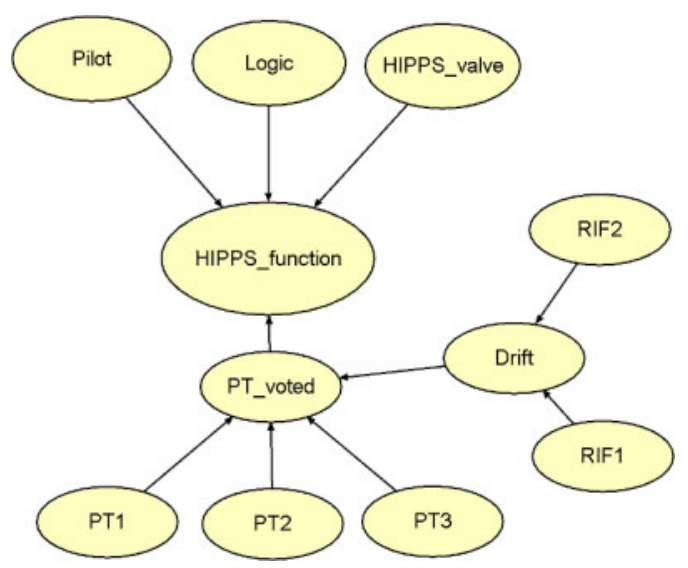

Figure 5. Two reliability influencing factors on the drifting.

\section{DISCUSSION AND CONCLUSION}

This paper use an illustrative example to demonstrate how to incorporate the foreseeable operational conditions of future subsea design (e.g. drifting of new subsea sensors) and how RIFs that in an early design phase can be foreseen as important, by the proposed reliability model that adapting Bayesian formalism. The presenting approach can provide an 'approximate but more closed to reality' indicator that reflects the best knowledge in the situation, to prove that the subsea system can operate as intended. The preliminary estimation can be continuously renewed through the evidence collection from the different stages of development (referred to the simulation in the early design phase).

The reliability model could be either very simple or very advanced, depends on modelling strategy. The prelimary proposal in this paper is not 'complete' and can be further improved, as it is subject to the following limitations and assumptions:

- The proposal can accomandate uncertainty involved in the novelty by improving the flexibility (by removing some restrictive assumptions) when model the system performance. The effect of data uncertainty (e.g. assigned value of RIFs) can be outlined by introducing probability distribution to variables. The level of completness uncertainty is still high because of, e.g. the proposal only provides a rather simple procedure that depends heavily on the element of judgement to determine the conditional probabilities between RIFs and failure cause (i.e. sensor drift). But the proposal is still promising as the level of uncertainty will be reduced within the increasing understanding of system risks and performance in the later phase. One promising approach is to provide an algorithm that combines the different type of data and relevance of the observed data in the suggested method. Some initiatives about identifying the relevance between systems (topside and subsea) have already been taken by Rahimi and Rausand (2013). The similar algorithm can be adapted in presenting method and even in a more advanced way due to the probabilistic characteristic of $\mathrm{BN}$ model.

- Considering the wear effect of subsea equipment is important since no preventive maintenance work are carried out subsea. Encountering Weibull distribution to present the increasing effect of degradation (e.g. drifting) in the suggested method is an area where further work needed.

- The presenting approach has not been implement against a real case. Our suggestion for further research work is to investigate the physics behind the sensor drift so that the realistic RIFs are selected. The sensitivity analysis should be performed to obtain the relative importance, the most important RIF can therefore selected to be included in the early evaluation of new subsea design.

\section{ACKNOWLEDGEMENT}

The authors gratefully thank to the support from the new center for innovation-driven research on Subsea Production and Processing (SFI SUBPRO). The author would also like to thank reviewers for giving comments to improve this research work.

\section{REFERENCES}

Bobbio, A., Portinale, L., Minichino, M., \& Ciancamerla, E. (2001). Improving the analysis of dependable systems by mapping fault trees into bayesian networks. Reliability Engineering and System Safety, 71, 249-260.

Brissaud, F., Charpentier, D., Fouladirad, M., Barros, A., \& Bérenguer, C. (2010). Failure rate evaluation with influencing factors. Journal of Loss Prevention in the Process Industries, 23, 187-193.

Cai, B., Liu, Y., Fan, Q., Zhang, Y., Yu, S., Liu, Z., \& Dong, X. (2013). Performance evaluation of subsea BOP control systems using dynamic Bayesian networks with imperfect repair and preventive maintenance. Engineering Applications of Artificial Intelligence, 26, 2661-2672.

Cai, B., Liu, Y., Liu, Z., Tian, X., Dong, X., \& Yu, S. (2012). Using Bayesian networks in reliability evaluation for subsea blowout preventer control system, 108, 32-41.

Durga Rao, K., Gopika, V., Sanyasi Rao, V. V. S., Kushwaha, H. S., Verma, A. K., \& Srividya, A. (2009). Dynamic fault tree analysis using Monte Carlo simulation in 
probabilistic safety assessment. Reliability Engineering \& System Safety, 94, 872-883.

HUGIN. (2015). software version $8.2 \mathrm{http}: / / \mathrm{www}$.hugin. com.

IEC61508. (2010). Functional safety of electrical/electronic/ programmable electronic safety related systems. Part 1-7. Geneva: International Electrotechnical Commission.

Innal, F. (2008). Contribution to modelling safety instrumented systems and to assessing their performance: Critical analysis of IEC 61508 standard, PhD Thesis. Bordeaux: University of Bordeaux

ISO/TR12489. (2013). Petroleum, petrochemical and natural gas industries - Reliability modelling and calculation of safety systems. Geneva: International Electrotechnical Commission.

Jensen, F. V. (1996). An introduction to Bayesian Networks. NJ, USA: Springer.

Jin, H., Lundteigen, M. A., \& Rausand, M. (2012). Uncertainty assessment of reliability estimates for safetyinstrumented systems. Proceedings of the Institution of Mechanical Engineers, Part O: Journal of Risk and Reliability, 226, 646-655.

Johansson, C. (2013). On system safety and reliability methods in early design phases, PhD thesis. Department of Management and Engineering, Linköping University, Linköping, Sweden.

Jones, B., Jenkinson, I., Yang, Z., \& Wang, J. (2010). The use of Bayesian network modelling for maintenance planning in a manufacturing industry. Reliability Engineering \& System Safety, 95, 267-277.

Khakzada, N., Khana, F., \& Amyotte, P. (2013). Dynamic safety analysis of process systems by mapping bow-tie into Bayesian network. Process Safety and Environmental Protection, 91, 46-53.

Long, D. (2012). A Primer For Model-Based Systems Engineering: Vitech Corporation.

Lundteigen, M. A., \& Rausand, M. (2010). Reliability of safety instrumented systems: Where to direct future research? Process Safety Progress, 29, 372-379.

MIL-HDBK-338B. (1998). Electronic Reliability Design Handbook: US Department of Defense.

Rahimi, M., \& Rausand, M. (2013). Prediction of failure rates for new subsea systems: A practical approach and an illustrative example. Proceedings of the Institution of Mechanical Engineers, Part O: Journal of Risk and Reliability August, 227, 629-640.

Rausand, M. (2014). Reliability of Safety-Critical Systems: Theory and Applications. Hoboken, NJ: Wiley.

Rausand, M., \& Høyland, A. (2004). System Reliability Theory, Models, Statistical Methods, and Applications (second edition ed.): John Wiley \& Sons, Inc.

SINTEF. (2010). Reliability Data for Safety Instrumented Systems - PDS Data Handbook, S.Hange, T.Onshus (Authors): SINTEF.

Vatn, J. (2013). Risk_OMT-Hybrid approach, Course PK8200-Risk Influence Modelling and Risk Indicators. Norway: NTNU. 